\title{
Reprodutibilidade de Laudos de uma mesma Manometria Segundo Diferentes Examinadores
}

\author{
Reproducibility of Manometric Test Results According to Different Examiners
}

\author{
MARIA AUXILIADORA PROLUNGATTI CESAR ${ }^{1}$; WILMAR ARTUR KLUG ${ }^{2}$; PERETZ CAPELHUCHNICK $^{3}$; JORGE \\ ALBERTO ORTIZ ${ }^{4}$; ANANDRÉA PIVA MANTOVANI ${ }^{5}$ CARLOS ANDRÉ DE BARROS ANTUNES ${ }^{6}$; RUY CHARLES \\ CARDOSO DE SOUZA ${ }^{7}$; CYNTIA DANIELA RESENDE DE VASCONCELOS ${ }^{8}$
}

\begin{abstract}
${ }^{1}$ Professora Doutora pela Faculdade de Ciências Médicas da Santa Casa de São Paulo, Assistente Colaborador III, Disciplina de Cirurgia do Departamento de Medicina da Universidade de Taubaté; ${ }^{2}$ Professor Titular Docente Livre do Departamento de Cirurgia da Faculdade de Ciências Médicas da Santa Casa de São Paulo; ${ }^{3}$ Professor Titular Docente Livre do Departamento de Cirurgia da Faculdade de Ciências Médicas da Santa Casa de São Paulo; ${ }^{4}$ Mestre em Cirurgia, Coordenador do Ambulatório de Fisiologia Anal da Faculdade de Ciências Médicas da Santa Casa de São Paulo; ${ }^{5}$ Ex-Residente do Departamento de Cirurgia da Faculdade de Ciências Médicas da Santa Casa de São Paulo; ${ }^{6}$ Ex-Residente de Coloproctologia, Departamento de Cirurgia da Faculdade de Ciências Médicas da Santa Casa de São Paulo: ${ }^{7}$ Ex-Residente de Coloproctologia, Departamento de Cirurgia da Faculdade de Ciências Médicas da Santa Casa de São Paulo; ${ }^{8}$ Ex-Residente de Coloproctologia, Departamento de Cirurgia da Faculdade de Ciências Médicas da
\end{abstract} Santa Casa de São Paulo - SP - Brasil.

CESAR MAP; KLUG WA; CAPELHUCHNICK P; ORTIZ JA; MANTOVANI AP; ANTUNES CAB; SOUZA RCC; VASCONCELOS CDR. Reprodutibilidade de Laudos de uma mesma Manometria Segundo Diferentes Examinadores. Rev bras Coloproct, 2007;27(1): 058-062.

RESUMO: Introdução: A manometria anal é um método para estudo do esfíncter anal, incluindo os músculos esfíncter anal interno e externo responsáveis pelas pressões de repouso e contração. Método: estudo retrospectivo através da análise de exames de manometria anal de 10 pacientes com diagnóstico de constipação atendidos no ambulatório de Fisiologia Anal do Serviço de Coloproctologia da Faculdade de Ciências Médicas da Santa Casa de São Paulo no período de janeiro de 2004 a julho de 2004. Todos os exames foram analisados por 3 diferentes examinadores sendo um preceptor em fisiologia anal, um médico em aprendizado recente e um médico residente em treinamento e cada um fornece um laudo para cada exame. Resultado: os examinadores são diferentes estatisticamente na avaliação da pressão de contração e de evacuação. Em algumas situações o examinador 1 é diferente dos outros examinadores e em uma situação o examinador 3 é diferente dos demais. Não houve diferença em relação aos laudos de presença de anismus, comprimento do canal anal, falha esfincteriana e laudo final do exame. Conclusão: diferenças estatísticas foram observadas entre os examinadores, porém, estas diferenças não modificaram o laudo final do exame. Seu resultado final não é influenciado pela reprodução do mesmo por diferentes profissionais.

Descritores: manometria, reprodutibilidade dos testes, aprendizagem, canal anal.

\section{INTRODUÇÃO}

Nas décadas de 1980 e 1990, diferentes testes foram desenvolvidos a fim de estudar a função anoretal. O mais comum e mais utilizado deles é a manometria anal que quantifica o tônus do esfíncter anal, avalia o reflexo inibitório anal e as pressões do canal anal, sendo utilizado com freqüência na investigação da constipação intestinal ${ }^{(1,2,3,4)}$.

A avaliação da função ano-retal consiste em medir os seguintes parâmetros:

1. Função do esfíncter anal, incluindo a pressão do esfíncter em repouso, contração e evacuação;

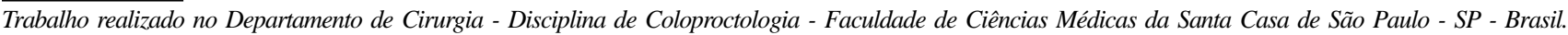


2. O comprimento funcional do canal anal;

3. Resposta da pressão anal após o aumento da pressão intra-abdominal;

4. Mudanças nas pressões anal e retal durante a tentativa de evacuação ${ }^{(1,2,3,4)}$.

Trabalhos anteriores estudaram a reprodutibilidade da manometria anal através do exame realizado com o mesmo grupo de pacientes, porém em períodos ou dias diferentes, seguindo a mesma técnica, e realizaram comparações entre exames realizados através de diferentes técnicas ${ }^{(5,6,7)}$.

O objetivo desse trabalho foi analisar a reprodutibilidade dos laudos da manometria anal em pacientes constipados através da interpretação dos mesmos exames por diferentes examinadores em diferentes níveis de aprendizado verificando os resultados das pressões de repouso, contração e evacuação e as conclusões finais do exame.

\section{PACIENTES E MÉTODOS}

Foi realizado estudo retrospectivo utilizando uma amostra de 10 pacientes do sexo feminino com diagnóstico de constipação intestinal, não chagásicos e não incontinentes, atendidos no ambulatório de fisiologia anal da disciplina de Coloproctologia da Faculdade de Ciências Médicas da Santa Casa de São Paulo no período de janeiro de 2004 a julho de 2004.

Todos os exames foram realizados seguindo a mesma técnica utilizando o aparelho Dynamed de perfusão com oito canais : sem preparo intestinal prévio, paciente em decúbito lateral esquerdo e a sonda introduzida até cinco centímetros acima do orifício anal com as medidas realizadas a cada centímetro (pressão máxima de repouso, pressão máxima de contração e pressão mínima de evacuação).

A amostra foi então avaliada por três diferentes examinadores, no mesmo dia e sem que houvesse troca de informações entre eles, sendo:

1. examinador 1: preceptor em fisiologia anal

2. examinador 2: médico em aprendizado recente

3. examinador 3: médico residente em treinamento

Os valores analisados a cada exame foram:

1. média de pressão máxima de repouso
2. média de pressão máxima de contração

3. média de pressão máxima de evacuação

A média máxima foi avaliada a cada centímetro, com intervalo considerado de $0,5 \mathrm{~cm}$ em relação à onda da manometria.

Interpretando esses valores, cada examinador responde às seguintes questões

1. se há presença de anismus

2. comprimento do canal anal

3. se há falha esfincteriana

4. se a manometria é normal ou anormal

Os resultados foram analisados pelo método programa SPSS (Statistical Package for Social Sciences), em sua versão 10.0, para a obtenção dos resultados. Adotamos o nível de significância de 5\% $(0,050)$.

Os dados foram analisados pelos testes de Friedman e Wilcoxon .

\section{RESULTADOS}

A aplicação do Teste de Friedman para identificação de possíveis diferenças entre os resultados dos três examinadores demonstrou os seguintes resultados:

No estudo das médias das pressões de repouso os três examinadores não apresentam diferenças estatisticamente significantes.(Tabela 1).

No estudo das médias das pressões de contração os três examinadores apresentam três diferenças estatisticamente significantes e duas estatisticamente não-significantes, como podemos observar na tabela 2 .

No estudo das pressões de evacuação os três examinadores apresentam uma diferença estatisticamente significante (a um centímetro do canal anal) e quatro estatisticamente não-significantes. (Tabela 3).

A aplicação do Teste dos Postos Sinalizados de Wilcoxon, para identificação de possíveis diferenças entre os pares de 'examinadores', nos quatro casos em que o Teste de Friedman apontou diferenças estatisticamente significantes demonstrou:

Na pressão de Contração a $5 \mathrm{~cm}$ o examinador 1 é estatisticamente diferente do examinador 3 como podemos observar na tabela 4 . 
Tabela 1- Médias das pressões máximas de repouso do canal anal a cada centímetro segundo os três examinadores.

\begin{tabular}{lcccc}
\hline Examinador / Altura & Examinador 1 & Examinador 2 & Examinador 3 & P \\
\hline $5 \mathrm{~cm}$ & 10,577 & 14,549 & 10,195 & 0,905 \\
$4 \mathrm{~cm}$ & 23,374 & 27,806 & 18,735 & 0,729 \\
$3 \mathrm{~cm}$ & 46,94 & 53,711 & 40,55 & 0,717 \\
$2 \mathrm{~cm}$ & 72,303 & 71,055 & 51,994 & 0,110 \\
$1 \mathrm{~cm}$ & 52,601 & 52,112 & 40,554 & 0,083 \\
\hline
\end{tabular}

Fonte-arquivo de exames de manometria anal do ambulatório de fisiologia anal da santa casa de São Paulo.

No estudo das médias das pressões de contração os três examinadores apresentam três diferenças estatisticamente significantes e duas estatisticamente não-significantes, como podemos observar na tabela 2.

Tabela 2 - Médias das pressões máximas de contração do canal anal a cada centímetro segundo os três examinadores.

\begin{tabular}{lcccc}
\hline Examinador / Altura & Examinador 1 & Examinador 2 & Examinador 3 & P \\
\hline $5 \mathrm{~cm}$ & 33,2725 & 23,83444 & 18,275 & 0,011 \\
$4 \mathrm{~cm}$ & 48,18125 & 42,60222 & 32,67 & 0,072 \\
$3 \mathrm{~cm}$ & 80,047 & 67,073 & 54,658 & 0,001 \\
$2 \mathrm{~cm}$ & 100,756 & 96,462 & 84,29 & 0,061 \\
$1 \mathrm{~cm}$ & 81,95 & 76,124 & 68,426 & 0,020 \\
\hline
\end{tabular}

Fonte - arquivo de exames de manometria anal do ambulatório de fisiologia anal da santa casa de São Paulo.

Tabela 3 - Médias das pressões mínimas de evacuação do canal anal a cada centímetro segundo os três examinadores.

\begin{tabular}{lcccc}
\hline Examinador / Altura & Examinador 1 & Examinador 2 & Examinador 3 & P \\
\hline $5 \mathrm{~cm}$ & 31,664 & 36,435 & 25,462 & 0,122 \\
$4 \mathrm{~cm}$ & 40,929 & 43,122 & 28,856 & 0,273 \\
$3 \mathrm{~cm}$ & 58,606 & 61,504 & 41,296 & 0,452 \\
$2 \mathrm{~cm}$ & 63,017 & 72,961 & 41,613 & 0,082 \\
$1 \mathrm{~cm}$ & 48,004 & 47,713 & 33,047 & 0,025 \\
\hline
\end{tabular}

Fonte - arquivo de exames de manometria anal do ambulatório de fisiologia anal da santa casa de São Paulo.

Na pressão de Contração a 3 e a 1 cm observamos que o examinador 1 é estatisticamente diferente dos demais como mostrado nas tabelas 5 e 6.

Na Pressão de Evacuação a $1 \mathrm{~cm}$ o examinador 3 é estatisticamente diferente dos demais (tabela 7).

Quando o estudo relacionou as conclusões dos exames os três examinadores apresentam diferenças estatisticamente não-significantes como observado na tabela 8 .

\section{DISCUSSÃO}

A manometria anal é um exame bastante utilizado, e dúvidas existem quanto ao fato dos resultados dos exames serem examinadores dependentes, já que é um estudo dinâmico. Além disso, não existe uma padronização mesmo nos diferentes serviços que possibilitem a confirmação da reprodutibilidade de resultados ${ }^{(1,6,7)}$.

Em estudo realizado sobre a reprodutibilidade inter e intra-individual da manometria anal de pacientes 
Tabela 4 - Comparação da pressão máxima de contração a $5 \mathrm{~cm}$ segundo os três examinadores.

\begin{tabular}{lcccc}
\hline & Examinador 1 & Examinador 2 & Examinador 3 & P \\
\hline Examinador1 vs Examinador 2 & 33,2725 & 23,83444 & & 0,123 \\
Examinador 1 vs Examinador 3 & 33,2725 & & 18,275 & 0,012 \\
Examinador 2 vs Examinador 3 & & 23,83444 & 18,275 & 0,314 \\
\hline
\end{tabular}

Fonte-arquivo de exames de manometria anal do ambulatório de fisiologia anal da santa casa de São Paulo.

Tabela 5 - Comparação da pressão máxima de contração a $3 \mathrm{~cm}$ segundo os examinadores.

\begin{tabular}{lcccc}
\hline & Examinador 1 & Examinador 2 & Examinador 3 & P \\
\hline Examinador1 vs Examinador 2 & 80,047 & 67,073 & & 0,018 \\
Examinador 1 vs Examinador 3 & 80,047 & & 54,658 & 0,005 \\
Examinador 2 vs Examinador 3 & & 67,073 & 54,658 & 0,333 \\
\hline
\end{tabular}

Fonte-arquivo de exames de manometria anal do ambulatório de fisiologia anal da santa casa de São Paulo.

Tabela 6 - Comparação da pressão máxima de contração a $1 \mathrm{~cm}$ segundo os examinadores.

\begin{tabular}{lcccc}
\hline & Examinador 1 & Examinador 2 & Examinador 3 & P \\
\hline Examinador1 vs Examinador 2 & 81,95 & 76,124 & & 0,037 \\
Examinador 1 vs Examinador 3 & 81,95 & & 68,426 & 0,037 \\
Examinador 2 vs Examinador 3 & & 76,124 & 68,426 & 0,203 \\
\hline
\end{tabular}

Fonte - arquivo de exames de manometria anal do ambulatório de fisiologia anal da santa casa de São Paulo.

Tabela 7 - comparação da pressão mínima de evacuação a $1 \mathrm{~cm}$ segundo os examinadores.

\begin{tabular}{lcccc}
\hline & Examinador 1 & Examinador 2 & Examinador 3 & P \\
\hline Examinador1 vs Examinador 2 & 48,004 & 47,713 & & 0,721 \\
Examinador 1 vs Examinador 3 & 48,004 & & 33,047 & 0,017 \\
Examinador 2 vs Examinador 3 & & 47,713 & 33,047 & 0,022 \\
\hline
\end{tabular}

Fonte - arquivo de exames de manometria anal do ambulatório de fisiologia anal da santa casa de São Paulo.

normais foi encontrado reprodutibilidade de resultados apenas nos parâmetros comprimento do canal anal e pressão máxima de repouso ${ }^{(5)}$. Resultado diferente foi encontrado em outra pesquisa com pacientes normais em que todos os parâmetros foram reprodutíveis ${ }^{(7)}$.

Neste estudo o que pudemos observar foi que apesar dos resultados numéricos serem diferentes entre os 3 examinadores, principalmente quando comparados preceptor e médico, com o residente em aprendizado, observamos que não houve diferença em relação à conclusão final dos exames. Isso é um dado muito importante encontrado, pois demonstra que o médico que recebe o exame não é dependente apenas da conclusão do médico examinador. Alguns trabalhos foram encontrados na literatura sobre a reprodutibilidade do exame ${ }^{(5,6,7)}$, nada encontramos sobre a reprodutibilidade dos laudos.

Outro dado importante está relacionado com a diferença encontrada quanto aos resultados numéri$\cos$ das pressões de repouso, contração e evacuação. Isso pode ser explicado, pois a escolha do intervalo medido fica por conta do examinador, e como as pressões são medidas em intervalos de mais ou menos 30 segundos, depende do examinador a escolha do inter- 
Tabela 8 - conclusão final dos exames de manometria anal segundo os 3 examinadores.

\begin{tabular}{ll}
\hline Diagnósticos & p \\
\hline Anismus & 0,097 \\
Comprimento do canal anal & 0,549 \\
Presença de falha esfincteriana & 0,368 \\
Exame normal/anormal & 0,368 \\
\hline
\end{tabular}

Fonte - arquivo de exames de manometria anal do ambulatório de fisiologia anal da santa casa de São Paulo. valo a ser medido, podendo o médico em aprendizado cometer erros neste momento.

\section{CONCLUSÃO}

$\mathrm{Na}$ avaliação das pressões de repouso, contração e evacuação, o preceptor é diferente do profissional em aprendizado, porém, em relação à conclusão do exame, não houve nenhuma diferença significativa em relação ao anismus, comprimento do canal anal, falha esfincteriana e laudo de conclusão final do exame.

\begin{abstract}
Introduction: The anal manometry is an approach for the study of the anal sphincter including the muscles internal and external anal sphincter being responsible for the pressures of rest and contraction. Method: retrospective study through the analysis of examinations of anal manometry of 10 patients with diagnosis of constipation taken care of in the clinic of Anal Physiology of the Service of Coloproctologia of the College of Medical Sciences of the Casa Saint of São Paulo in the period of January of 2004 the July of 2004. All the examinations had been analyzed by 3 different examiners being a teacher in anal physiology, a doctor in recent learning and a resident doctor in training and each one supply a finding each examination. Result: the examiners are different statistical in the evaluation of the evacuation and contraction pressure. In some situations examiner 1 is different of the other examiners and in a situation examiner 3 is different of excessively. It did not have difference in relation to the findings of presence of anismus, length of the anal canal, final finding of the examination fails esfincteriana and. Conclusion: statistical differences had been observed between the examiners, however, these differences had not modified the final finding of the examination. Its final result is not influenced by the reproduction of it by different professionals.
\end{abstract}

Key words: manometry, learning, reproducibility of results, anus, constipation, anal canal.

\section{REFERÊNCIAS}

1. Sun WM, Rao SS: Manometric assessment of anorectal function. Gastroenterol Clin North Am 2001; 30: 15-32.

2. Ashraf W, Park F, Lot J,Quigley EM: An examination of the Reliability of reported stool frequency in the diagnosis of idiopathic constipation. The Am J Gastroenterol 1996; 91 : 26-31.

3. Halverson AL, Orkin BA: Which Physiologic Tests Are Useful in Patients with Constipation? Dis Colon Rectum 1998; 41 : 735-738.

4. Schäfer R, Heyer T, Gantke B, Schäfer A, Frieling T,Haussiner D, Enck P: Anal endosonography and manometry - comparison in patients with defecation problems. Dis Colon Rectum 1997; $40: 293-296$.

5. Freys SM, Fuchs KH, Fein M, Heimbucher J, Sailer M, Thiede A: Inter and intraindividual reproducibility of anorectal manometry .Langenbecks Arch Surg.1998; 383: 325-329.
6. Bharucha AE, Seide B, Fox JC, Zinsmeister AR: Day-to-day reproducibility of anorectal sensorimotor assesments in healthy Subjects. Neurogastroenterol Motil 2004; 16(2): 241250.

7. Cesar M.A.P. ; Ortiz , J.A. ; Faria ,F.G. ; Salgado ,F.C. ; Salan, F.O.; Branco ,G.A.R. ; Sayegh ,K. ;Neder ,R.P. ; Bassi , D.G.; Speranzini , M.B. Reprodutibilidade da manometria anal em mulheres sem distúrbios evacuatórios. Rev Bras Coloproctol, 2004; 24(1) : 33-37.

Endereço para correspondência: MARIA AUXILIADORA PROLUNGATTI CESAR

Av. Juscelino K de Oliveira, 530 / apto. 51

Taubaté - SP

12010-600

FAX: (12) 3629-1079

e-mail: prolungatti@uol.com.br 